\title{
Consequences to the Lungs When Gas Swings Between Lung Units During Patient Triggered Mechanical Ventilation
}

In this issue of the Journal, Enokidani et $\mathrm{al}^{1}$ studied the impact of ventilator settings on the extent of pendelluft during mechanical ventilation using a lung simulator model. Pendelluft was first described in patients with chronic lung disease, in whom it can occur during inspiration or expiration, in spontaneous breathing as well as in controlled ventilation., ${ }^{2,3}$ Initially, pendelluft was defined as the movement of gas from one lung unit to another (ie, air movement within the lungs) during either inspiration or expiration. ${ }^{2}$ More recently, pendelluft has been detected in patients with ARDS before initiation of the breath, in the absence of tidal volume changes. In the presence of acute lung injury, vigorous spontaneous breathing effort causes a pendelluft phenomenon, with redistribution of air from nondependent to dependent lung regions, following different regional compliance properties of the lungs. ${ }^{3}$ This movement results in overdistention of dependent lung and has the potential to cause patient-induced lung injury.

Pendelluft can occur during patient triggered mechanical ventilation or spontaneous breathing, but typically does not occur during controlled ventilation, except in patients with chronic lung disease..$^{3-5}$ Pendelluft is also considered one of the mechanisms that explain gas transport during highfrequency jet ventilation or high-frequency oscillation. ${ }^{6}$ Pendeluft may be marked when mechanical ventilation is used in a patient with heterogeneous impedances. ${ }^{7,8}$

The presence of pendelluft can be identified with auscultation of the chest or, more practically and definitively, with the use of electrical impedance tomography (EIT). Figure 1 illustrates an EIT image of a swine model of severe ARDS during controlled and patient triggered mechanical ventilation. During controlled ventilation, the impedance changes are synchronous in the ventral and dorsal regions of the lungs during a respiratory cycle. Once the ventilator is switched to assisted mechanical ventilation, the pendelluft phenomenon appears. In the early phases of inspiration, gas moves within the lungs from the nondependent regions of the lungs to the most dependent. With vigorous inspiration, the pleural

The authors have disclosed no conflicts of interest.

Correspondence: Robert M Kacmarek PhD RRT FAARC, Department of Respiratory Care, Massachusetts General Hospital, 55 Fruit St, Warren 1225, Boston, MA 01460. E-mail: rkacmarek@mgh.harvard.edu.

DOI: $10.4187 /$ respcare. 08764 pressure changes in the dependent area of the lung are greater than the pleural pressure changes of the nondepend-

\section{See the Original Study on Page 1}

ent lung. The atelectasis prevents the equalization of the dependent and nondependent pleural pressure because pressure does not transmit simultaneously through the atelectatic lung during inspiration, creating a pressure gradient between nondependent and dependent lung. As a result, gas moves from the nondependent lung into the dependent lung during inspiration. In this scenario, patients may develop significant flow asynchrony and, potentially, self-inflicted lung injury.

In 2013, Yoshida et $\mathrm{al}^{3}$ published the effects of flow asynchrony, during both patient triggered volume and pressure ventilation, on the development of pendelluft in patients and animals with severe acute hypoxemic respiratory failure. Figure 2 nicely illustrates the fading of pendelluft during transition from spontaneous breathing to controlled ventilation via paralysis obtained by intravenous bolus of succinylcholine chloride in an animal model of severe hypoxemic respiratory failure. During spontaneous breathing with the initiation of inspiration, gas moves from the nondependent lung regions (Zone 1 in the figure) to the most dependent regions (Zone 3 in the figure), increasing the dependent tidal volume, which subsequently normalizes after initiation of paralysis. As a result, in spontaneous breathing the dependent lung is overinflated during inspiration while the nondependent lung has a minimal, if any, tidal volume, increasing the potential of patient-induced lung injury.

Enokidani et al $^{1}$ determined that ventilator settings have no marked impact on the level of pendelluft. They used 2 sets of twin-bellows training test lungs in which 1 bellows in each set acted as the lung, while the other served as the mechanism used to generate inspiratory effort. The generated inspiratory effort triggered the ventilators attached to the lung model. They studied the effects of ventilatory mode (ie, volume and pressure assist/control), trigger sensitivity (ie, flow-triggering 1, 2, 3, 4, and $6 \mathrm{~L} / \mathrm{min}$ ), inspiratory pressurization $(1 \%, 25 \%, 50 \%, 75 \%, 100 \%$ pressure assist/control), and inspiratory cycling-off criteria (they used 6 rise times to affect cycling criteria: $0.6,0.8,1.0,1.2$, 1.4 , and $1.6 \mathrm{~s}$ ). They used one set of respiratory mechanics. In the normal nondependent lung, the compliance was 40 $\mathrm{mL} / \mathrm{cm} \mathrm{H}_{2} \mathrm{O}$, and the resistance was $5 \mathrm{~cm} \mathrm{H}_{2} \mathrm{O} / \mathrm{L} / \mathrm{s}$; in the 


\section{EDITORIALS}

\section{Controlled Mechanical Ventilation}

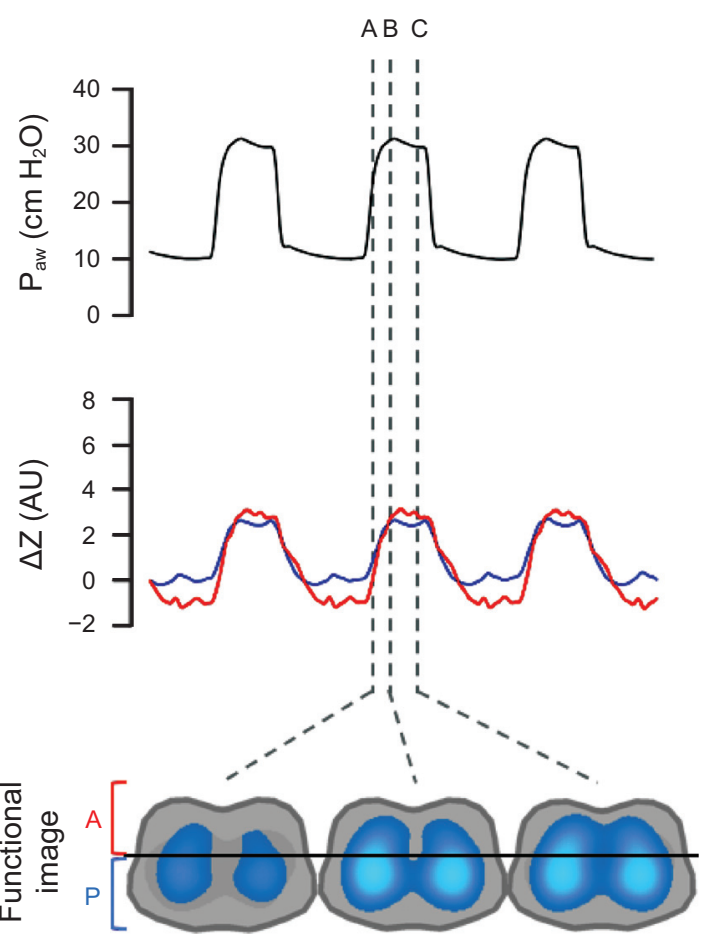

Assisted Mechanical Ventilation

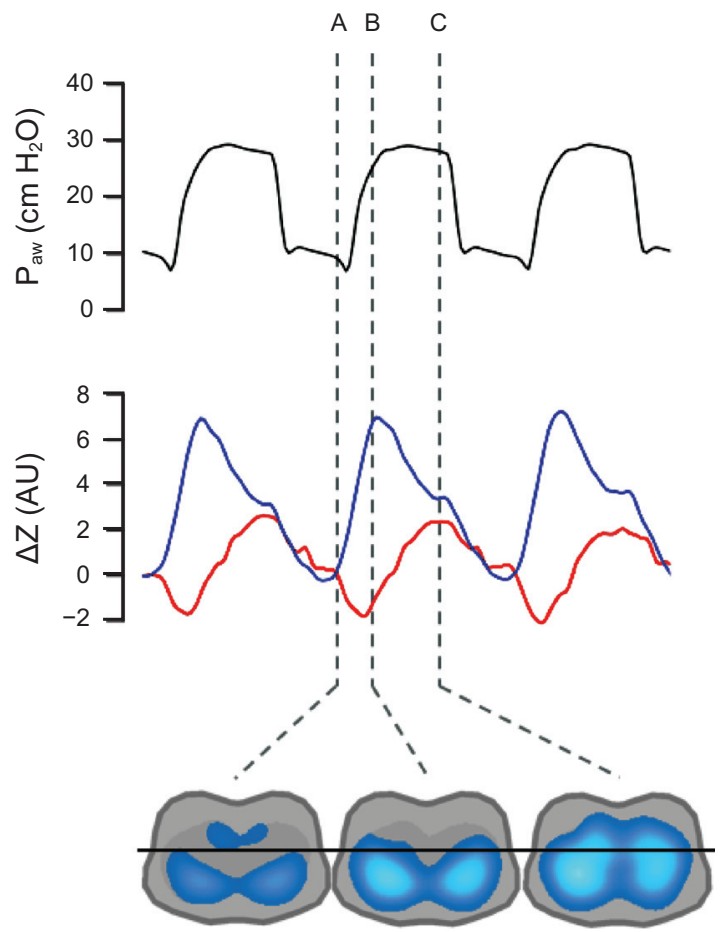

Fig. 1. Airway pressure $\left(P_{a w}\right)$, variation of impedance $(\Delta Z)$, and electrical impedance tomography images in controlled and assisted mechanical ventilation demonstrating pendelluft phenomenon in an experimental model with severe ARDS. Blue line: posterior regions of lung. Red line: anterior regions of lung. During controlled mechanical ventilation, the impedance changes in the ventral and dorsal regions of the lungs are synchronous during a respiratory cycle. During assisted mechanical ventilation, the impedance of the anterior regions of lung are decreased (eg, loss of gas in the air space) whereas the impedance in increased in the posterior region (eg, increased gas in the air space). AU =arbitrary units. Figure courtesy of Caio C. A. Morais.

dependent lung, the compliance was $10 \mathrm{~cm} \mathrm{H}_{2} \mathrm{O}$ and the resistance was $20 \mathrm{~cm} \mathrm{H}_{2} \mathrm{O}$. Five different pleural pressures were applied to the dependent region of the lung ranging from none (controlled ventilation) to very negative.

We congratulate Enokidani et $\mathrm{al}^{1}$ for providing us with insights on the effects of some ventilatory variables on the development or extension of pendelluft. However, there are some concerns regarding the study design. First, it was performed on a lung model and not on patients. It is doubtful that any institutional review board would approve such a study. The positives of a lung model study are the fact that it can focus on numerous variables over a wide range of values. Lung model studies also provide data to develop clinical studies focused more narrowly on a specific aspect that can be studied in patients. Second, it would be more insightful if actual modification of cycling criteria occurred as opposed to using rise time adjustments as a replacement of cycling criteria. Third, a number of variables that could affect pendelluft level were not studied. Specifically, an increased number of compliance and resistances settings could have been studied, as well as various breathing frequencies, tidal volumes, and PEEP levels.
In closing, we should remember that pendelluft is the movement of gas within the lungs (that might occur at any time in the breathing cycle) from one lung unit of low impedance to another lung unit with higher impedance due to marked differences in local pleural pressure. This results in overdistention of the dependent lung and a high potential for the development of patient-induced lung injury. Pendelluft is a phenomenon observed not only in patients with chronic pulmonary disease but increasingly in patients with severe acute hypoxemic respiratory failure and dependent atelectasis, and in patients with consolidated lung units causing pleural pressure differences between dependent and nondependent lung in the presence of flow asynchrony. Although there is no specific approach to minimizing pendelluft, attention should be paid to flow asynchrony and modifying gas delivery to avoid asynchrony. If asynchrony cannot be corrected and the patient is at increased risk of lung injury, the patient should be sedated and administered controlled ventilation. Clinically, the only way to specifically identify the presence of pendelluft at the bedside of intubated patients at the present time is the use of EIT, as shown in Figure 1 and Figure 2. 


\section{EDITORIALS}

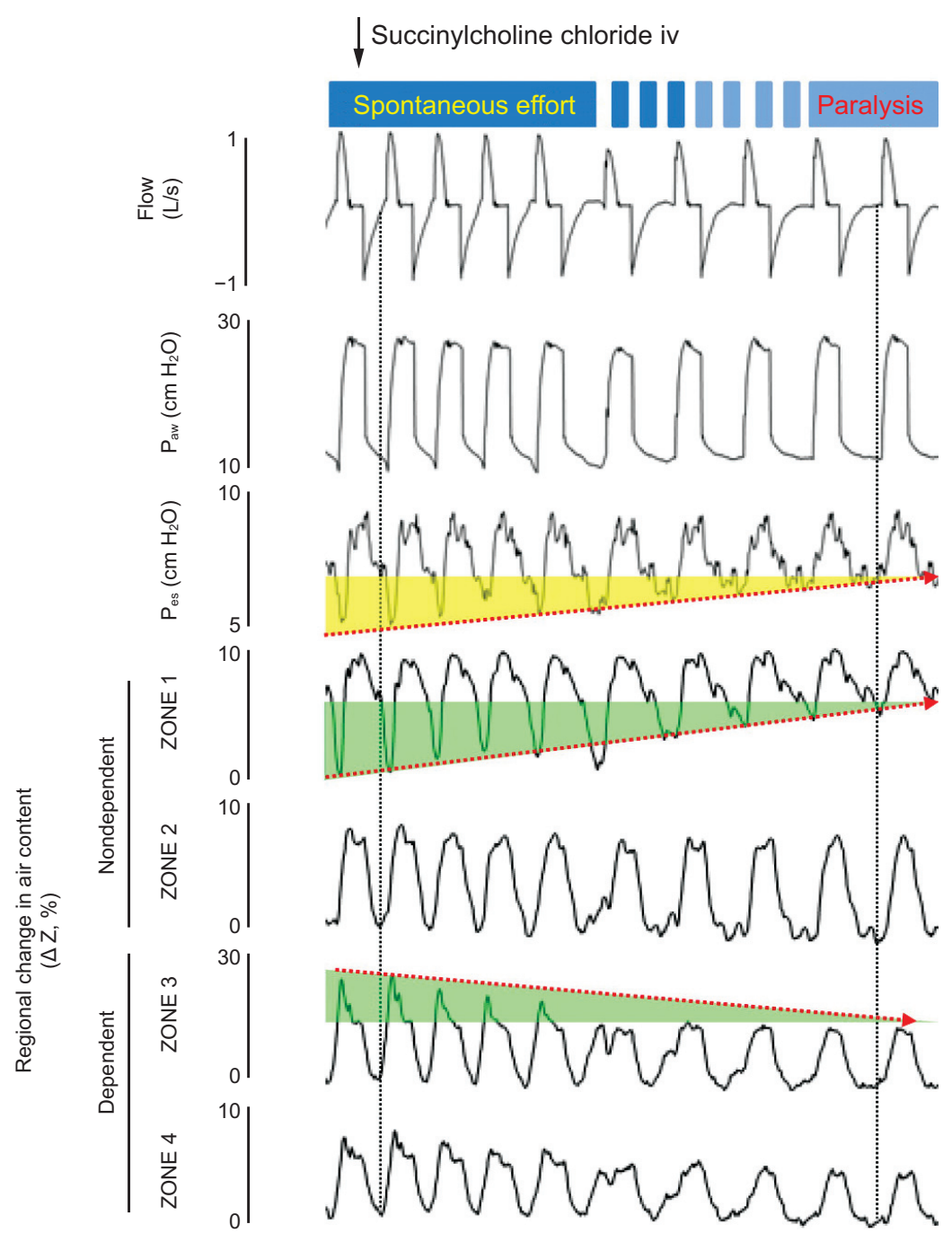

Fig. 2. Electrical impedance tomography waveforms in transition from spontaneous breathing to muscle paralysis. After injection of succinylcholine chloride, negative deflections in esophageal pressure were reduced (yellow) as paralysis developed. As spontaneous breathing effort weakened, the degree of pendelluft became smaller (green). $P_{a w}=$ airway pressure; $P_{e s}=$ esophageal pressure; $\Delta Z=$ variation of impedance. From Reference 3, with permission.

Robert M Kacmarek Lorenzo Berra Department of Respiratory Care Massachusetts General Hospital Boston, Massachusetts

Jesús Villar

CIBER de Enfermedades Respiratorias Instituto de Salud Carlos III Madrid, Spain

\section{REFERENCES}

1. Enokidani Y, Uchiyama A, Yoshida T, Abe R, Yamashita T, Koyama Y, Fujino Y. Effects of ventilatory settings on Pendelluft phenomenon during mechanical ventilation. Respir Care 2021;66(1):1-10.

2. Beydon L, Svantesson C, Brauer K, Lemaire F, Jonson B. Respiratory mechanics in patients ventilated for critical lung disease. Eur Respir $\mathrm{J}$ 1996;9(2):262-273.
3. Yoshida T, Torsani V, Gomes S, De Santis RR, Beraldo MA, Costa EL, et al. Spontaneous effort causes occult pendelluft during mechanical ventilation. Am J Respir Crit Care Med 2013;188(12): 1420-1427.

4. Alzahrany M, Banerjee A. A biomechanical model of pendelluft induced lung injury. J Biomech 2015;48(10):1804-1810.

5. Tabuchi A, Nickles HT, Kim M, Semple JW, Koch E, Brochard L, et al. Acute lung injury causes asynchronous alveolar ventilation that can be corrected by individual sighs. Am J Respir Crit Care Med 2016;193(4):396-406.

6. Chang HK. Mechanisms of gas transport during ventilation by high frequency oscillation. J Appl Physiol Respir Environ Exercise Physiol 1984;56(3):553-563.

7. Venegas JG, Tsuzaki K, Fox BJ, Simon BA, Hales CA. Regional coupling between chest wall and lung expansion during HFV: a positron imaging study. J Appl Physiol (1985) 1993;74(5):2242-2252.

8. Greenblatt EE, Butler JP, Venegas JG, Winkler T. Pendelluft in the bronchial tree. J Appl Physiol (1985) 2014;117(9):979-988. 